\title{
émulations
}

\section{Étienne Balibar - Europe. Crise et fin ?}

\section{Arthur Duhé}

Émulations - Revue de sciences sociales

2018, «Comptes rendus critiques, En ligne»

\section{Article disponible à l'adresse suivante}

https://ojs.uclouvain.be/index.php/emulations/article/view/7403

\section{Pour citer cet article}

Arthur Duhé, «Étienne Balibar — Europe. Crise et fin ? ", Émulations, en ligne. Mise en ligne le 4 avril 2018.

DOI : 10.14428/emulations.cr.040

Distribution électronique : Université catholique de Louvain (Belgique) : ojs.uclouvain.be

(C) Cet article est mis à disposition selon les termes de la Licence Creative Commons Attribution, Pas d'Utilisation Commerciale 4.0 International. http://creativecommons.org/licenses/by-nc/4.0/

Éditeur : Émulations - Revue de sciences sociales / Presses universitaires de Louvain https://ojs.uclouvain.be/index.php/emulations

ISSN électronique : 1784-5734

UCL PRESSES 


\section{Étienne Balibar - Europe. Crise et fin ?}

Arthur Duhé 1

Recensé : Étienne Balibar, Europe. Crise et fin?, Lormont, Le Bord de l’eau (« Diagnostics»), 2016, $322 \mathrm{p}$.

Cet ouvrage d'Étienne Balibar rassemble des interventions données entre 2010 et 2015, regroupées autour de divers thèmes tels que la montée du populisme, la mondialisation, la réforme de l'Europe, la question de la frontière ou encore celle des réfugiés qui pose en creux celle de la citoyenneté européenne. Ces interventions mêlent également des tons différents, entre rigueur philosophique et discours politique pour une audience plus large. Dans l'organisation même de l'ouvrage, l'auteur s'attache donc à remettre en cause le singulier qu'il a pourtant choisi pour son titre. En effet, Balibar montre ici la pluralité des crises que traverse l'Europe : crise du Grexit qui révèle la confrontation entre plusieurs Europe, l'Europe du Nord et celle du Sud, dont les intérêts nationaux sont divergents sinon opposés ; crise démocratique également puisque les institutions européennes souffrent, à juste titre selon l'auteur, d'un déficit de légitimité; crise du projet européen et de la notion même de peuple européen ; crise des réfugiés, enfin, qui exacerbe les tensions entre les États européens.

La diversité des périodes d'écriture permet ainsi de suivre l'actualité de l'Union européenne au cours des cinq dernières années, tout en la concentrant autour de quelques problématiques centrales. Revers de la médaille, certains discours ou articles sont maintenant datés tant l'actualité européenne a été chargée au cours des dernières années (le Brexit étant sans doute l'événement fondamental des derniers mois). Cette distinction des niveaux de crise permet cependant à l'auteur de maintenir un discours nuancé, blâmant ici la Chancelière allemande, Angela Merkel, pour ses positions dans la crise grecque mais rendant hommage à sa décision d'accueillir les réfugiés.

En intitulant son avant-propos interregnum, Balibar témoigne de son désir d’interroger la décomposition en cours de l'ordre européen, afin de dégager les différents horizons qui s'ouvrent devant ce dernier. Le titre trouve ici sa justification : si l'Europe traverse à l'évidence une période trouble, il est difficile pour l'heure d'en prédire l'issue. S’il se refuse à tout optimisme qui voudrait voir dans le délitement de l'ancien monde la certitude d'un ordre nouveau exempt des défauts de son prédécesseur, Balibar affirme néanmoins à plusieurs reprises que le pire n'est pas toujours sûr, et qu'une autre Union européenne est envisageable.

${ }^{1}$ Doctorant en relations internationales à l'Université d'Oxford, ancien élève de l'École normale supérieure de la rue d'Ulm. 
L'organisation de l'ouvrage en une diversité de perspectives qui, tantôt se juxtaposent, tantôt se complètent, nous interdit d'être exhaustif. Nous nous intéresserons donc ici plus particulièrement à la question du manque de légitimité démocratique dont souffre l'Union européenne, et dont nous exposerons brièvement les raisons. Il nous semble en effet que cette crise, qui interroge la nature de l'identité de l'Union européenne autant que son projet, permet également d'éclairer d'un jour nouveau tout à la fois les autres crises (celle du projet européen, celle de l'économie, celle des réfugiés, etc.) et les solutions possibles. C'est précisément à ces solutions, à la réponse que l'on peut faire à ce manque de légitimité, que nous consacrerons la seconde partie de ce compte-rendu.

L'Union européenne s'est construite à travers l'opposition idéologique entre un discours souverainiste, d'une part, entretenant «l'idée de communautés nationales naturelles ou "naturalisées" par l'histoire, auxquelles il serait toujours possible d'en revenir pour fonder la légitimité des institutions sur l'expression de la volonté générale » et un discours fédéraliste, d'autre part, avançant «l'idée d'un demos européen virtuel [...] du fait qu'il existe une structure représentative supranationale » (p. 110). Or le système européen est déjà mixte, puisque plus fédéral qu'on le croit et moins démocratique qu'il le prétend, n'ayant que peu de contre-pouvoirs effectifs. Surtout, ce système déjà instable et qui se heurtait à des oppositions ${ }^{2}$ se voit davantage déstabilisé en raison de l'émergence d'une nouvelle institution, à savoir la Banque centrale. Ce nouvel acteur dans le jeu européen reflète pour l'auteur « l'époque où le pouvoir politique ne se sépare plus du pouvoir économique et surtout financier » (p. 111). Selon Balibar, ce nouveau phénomène «ne reflète ni le simple développement de la technocratie ni seulement l'emprise du capitalisme privé », mais bien " une tentative de "révolution par en haut" " (p. 111).

Cependant, les nouveaux pouvoirs octroyés à la Banque centrale pour défendre la monnaie unique et réguler, de façon limitée, le marché financier, n'ont guère conféré à l'Union européenne plus de légitimité, et les dirigeants nationaux se sont alors présentés comme les seuls représentants de la souveraineté populaire. En conséquence, la légitimité démocratique se retrouve rognée au niveau supranational comme national. On retrouve ici un processus sur lequel Balibar insiste à plusieurs reprises dans l'ouvrage : en refusant la théorie des vases communicants qui voudrait que la légitimité démocratique pourrait se déplacer du niveau national au niveau supranational, et inversement, l'auteur formule "une "loi" de décomposition simultanée des procédures démocratiques au niveau national et au niveau européen » (p. 209). Plus encore, il affirme que «la construction postnationale n'a de chance de faire face à des secousses aussi violentes que les crises actuelles que si elle offre à tous les citoyens européens la

${ }^{2}$ On pense ici au rejet du projet du traité constitutionnel en 2005 par la France, les Pays-Bas et l'Irlande. 
perspective d'un progrès démocratique et non la préservation a minima de la démocratie partielle (plus ou moins oligarchique) dont ils jouissent jusqu'à présent» (p. 210). Cette loi, qu'il oppose à la théorie des vases communicants, s'inspire d'un principe spinoziste, à savoir : «l'accroissement simultané (et combiné) de la puissance d'agir aux différents niveaux» (p. 298).

Dans sa recherche de solutions à cette crise, l'auteur présente un point de vue qu'il reconnaît «européiste de gauche» (p. 275). Cette expression qui, de son aveu même, peut sembler maintenant contradictoire, étant donné le désamour de la gauche radicale pour le projet européen, exprime néanmoins la nécessité de construire une autre Europe qui s'opposerait à celle, néo-libérale, prise dans ses contradictions. Pour décrire cette Europe de gauche, Balibar recourt à une série de qualificatifs dont, par exemple, «Europe sociale » ou «Europe démocratique ». La défense d'une autre vision de ce projet européen, plutôt qu'un retour à des nations divisées, repose sur l'interprétation selon laquelle la construction européenne est irréversible car les conséquences d'un repli national seraient « désastreuses» (p. 279). On regrettera néanmoins ici un argumentaire quelque peu elliptique puisqu'il est difficile de voir en quoi le fait que la déconstruction européenne mènerait à un désastre (mais lequel ?) l'empêcherait de se produire. Surtout, cet argumentaire, qui date d'août 2015, ne peut être reçu qu'avec recul sinon suspicion après le vote en faveur du Brexit.

Pourtant, cette autre Europe sera le fruit d'une lutte entre deux camps extérieurs aux institutions de l'Union européenne, à savoir, d'un côté, le marché, et, de l'autre, «les masses de "citoyens passifs" refoulés en dehors des discussions sur le système politique et social» (p. 283). Or, on observe partout en Europe des multitudes se rassembler et protester contre l'ordre européen. La lutte se fera sur trois fronts : « [les] alternatives à l'austérité, [l] insertion de l'Europe et des nations européennes dans la mondialisation, [la] dérive antidémocratique des institutions » (p. 284). À l'austérité, nous devons donc substituer un système de répartition des ressources au sein de l'Union européenne et une construction budgétaire commune, une « union de transfert » (p. 286), qui viserait une transition de l'ensemble de l'Europe vers de nouvelles industries et de nouvelles formes d'énergies. Ensuite, il semble nécessaire que l'Europe se fasse altermondialisatrice, c'est-à-dire qu'elle «change le mode de mondialisation du monde», notamment en s'opposant à l'évasion fiscale ou aux ventes d'armes. Enfin, il faut inventer une démocratie transnationale; l'échec de Syriza ayant, selon Balibar, permis de constater l'état de délabrement démocratique atteint par l'Union européenne. Pour l'auteur, la démocratie n'est jamais réalisée absolument mais doit sans cesse être en progrès, et constitue donc un processus dynamique sans fin. Nous retrouvons ici l'idée que la démocratisation de l'Union européenne ne peut se faire que si elle est également accompagnée par une démocratisation à léchelle nationale. Si le changement de paradigme (des vases communicants vers le double processus de démocratisation) est majeur, il est à déplorer qu'il est parfois difficile de savoir ce que Balibar a précisément à l'esprit quand il appelle de ses vœux cette double démocratisation. La raison de ce flou est peut- 
être qu'il n'y a pas ici de réponse directe aux théories de l'intégration européenne élaborées dans le champ des sciences politiques. En revanche, on pourra lire dans cet ouvrage un dialogue critique avec Jurgen Habermas, autre philosophe s'intéressant de près à l'Union européenne (Habermas, 2012). En effet, en réponse au penseur de Francfort et à son optimisme vis-à-vis d'un possible fédéralisme européen, l'auteur souligne l'ampleur de la crise, laquelle est déjà bien installée dans les divers états qui composent l'Union européenne. Par ailleurs, le philosophe allemand, en privilégiant une démocratie formaliste et donc la représentation parlementaire, occulte le fait que la démocratie ne pourra venir que de revendications populaires diverses. Autrement dit, pour l'auteur, si la démocratie parlementaire est bien une part du projet démocratique, elle ne saurait se faire sans une démocratie conflictuelle, venue d'en bas et poussée par l'indignation.

Si donc la lutte pour une autre Europe est possible, elle suppose l'existence d'un "parti de l'Europe» (p. 306) qui resterait un mouvement libre, préservant une certaine diversité interne, et dès lors s'écarterait de la forme traditionnelle du parti. Même si Balibar n'y fait pas directement référence ici, on peut penser aux expériences des Indignados ou de Podemos qu'il mentionne quelques pages auparavant ou, plus récemment, à celle de la France insoumise. On le voit, l'auteur quitte ici la description pour entrer dans un discours plus normatif ou, pour le dire autrement, plus engagé politiquement. Nous regrettons de ne pas voir Balibar faire ici de liens explicites avec ses propres travaux portant, en référence à Spinoza, sur le concept de «multitude » (Balibar, 1985a et b). Il est évident que ces recherches ont, d'une certaine façon, influencé l'auteur, puisque nous retrouvons sous sa plume les termes de «multitude» (p. 49), d'«imperium» (p. 61) ou même d'«indignation ». Une approche plus frontale de ces thèmes aurait permis de penser l'ambiguïté importante des mouvements anti-européens qui regroupent tant des protestations nationalistes, voire xénophobes, que d'autres de gauche radicale ou d'extrême-gauche. C'est en effet un des sujets d'étude importants des marxistes spinozistes aujourd'hui que d'analyser ce que l'on peut espérer de la multitude ou des masses, entre optimisme révolutionnaire et pessimisme pragmatique, sujet que Balibar a largement contribué à constituer. Europe. Crise et fin ? aurait dès lors pu être l'occasion d'une confrontation entre la théorie spinoziste et une réalité contemporaine, éprouvant ainsi la théorie et éclairant la réalité.

\section{Bibliographie}

BALIBAR É. (1985a), «Spinoza, l'anti-Orwell. La crainte des masses», Les Temps modernes, vol. 41, $n^{\circ}$ 470, p. 353-398.

Balibar É. (1985b), Spinoza et la politique, Paris, Presses universitaires de France.

HABERMAS J. (2012), La Constitution de l’Europe, Paris, Gallimard. 